\title{
Teenagers, screens and social media: a commentary on Orben's narrative review
}

\author{
Manuela Ferrari ${ }^{1} \cdot$ Anita Schick ${ }^{2}$
}

Received: 30 January 2020 / Accepted: 4 March 2020 / Published online: 6 May 2020

c) Springer-Verlag GmbH Germany, part of Springer Nature 2020

As stated by Paracelsus centuries ago: "Poison is in everything, and no thing is without poison. The dosage makes it either a poison or a remedy." This maxim, deeply embedded in common sense, has also influenced risk assessment in many areas. For instance, the growing use of digital technologies over the past decades by children and adolescents has led to concerns among parents, educators and health providers for their potentially negative, or "poisonous", impact. Their concerns, echoed by those of researchers who also tend to view digital technology use as problematic, focus on the consequences of digital technology for health and well-being but most especially for the mental health of young people at a sensitive phase of development. Seeking to establish much needed scientific evidence may increase understanding on this topic, while guiding policy and decision-making for the benefit of everyone concerned about the use of digital technology among youth.

In this context, the publication of Amy Orben's review of reviews on the association between digital technologies and adolescent psychological well-being in Social Psychiatry and Psychiatric Epidemiology is important and timely [1]. Based on the work of Dickinson and colleagues [2] the Orben review included only high-quality studies involving the association between screen time, i.e. the amount of time spent interacting with screens, social media use and

This comment refers to the article available at https://doi. org/10.1007/s00127-019-01825-4.

Manuela Ferrari and Anita Schick contributed equally to this commentary.

Manuela Ferrari

manuela.ferrari@douglas.mcgill.ca

1 Department of Psychiatry, McGill University, Montreal, Canada

2 Department of Public Mental Health, Central Institute of Mental Health, Medical Faculty Mannheim, Heidelberg University, Mannheim, Germany adolescent mental health. In this commentary, we argue that the relationship between digital technology, specifically screen time, and the mental health of young people needs to be further understood in relation to possible outcomes, by focusing on the "interaction" between users and their devices within both their natural environment and the larger sociocultural context.

One of the greatest challenges facing researchers interested in understanding the impact of digital technology use on adolescent mental health concerns how to define "the problem" and the selection of appropriate variables for study. As Orben found, most studies are based on an overly simplistic definition of screen time, as the amount of time a user spends interacting with screens within a specific time frame. This conceptualization is, in our view, incomplete, since screen time involves a range of interactions with digital technology and activities such as watching, reading, browsing or interacting on social media and gaming. Social media use, as screen time activity involves a variety of actions that may be used in either active or passive ways.

Given the multiplicity of factors involved in digital technologies and different patterns of use, it is not surprising that the results of previous studies on the association between digital technology use (i.e. screen time and time spent on social media) and mental health have been mixed or that negative perceptions persist. Both Orben and Dickinson have reported a lack of clear evidence on factors that may potentially mediate and/or moderate the relationship between screen-based activities and mental health outcomes; nor has this relationship been sufficiently contextualized [2]. As well, very few study samples have included subsets of populations (e.g. specific age groups) or controlled for group differences on variables such as gender or mental health status, leaving many unanswered questions about the impact of screen time on child development or on groups with specific characteristics [2].

Defining the research problem is crucial for determining not only possible study outcomes, but also which research 
method or methods should be employed. In this connection, it should be noted that the digital revolution has drastically transformed human interaction with digital devices [3]. No longer passive consumers in front of a screen, modern users, including children and adolescents, are living a new reality as active participants interacting with others, selecting content, and shaping their digital experience by customizing their applications and contributing to the creation of new digital content [3, 4]. For example, young players interacting with digital games seek to discover new environments, learn new skills, overcome challenges and more, competing but also spending time with their peers [5, 6]. Digital game players are very active, both cognitively and emotionally, during their gaming experience. At the same time, action, sports or other games activate very different responses and learning experiences in players. Different activities using digital technologies are often performed in parallel, like chatting with a friend while watching a video [3].

This rich and creative relationship between users and their screens poses challenges for the development of research questions, not least the key question of whether user interaction is understood with enough complexity. Digital game is an example, when user-screen interaction is perceived as unidimensional, as if players were spending time passively in front of a screen and gaming were a solitary activity, researchers tend to generate studies that focus on the negative impact of video games, describing their risk potential for aggression, violence, and addiction [7, 8]. This unidimensional and narrow understanding of screen time and game play generates studies that focus mainly on the associations between gaming and mental illness (e.g., depression, anxiety, ADHD symptoms, and hostility/aggression) and, less frequently, on psychosocial wellbeing (e.g., social support/skills, loneliness). Among the eight reviews assessed by Dickinson, all of them classified as low quality studies, anxiety and depression were the mental health outcomes most frequently measured. Similar arguments could be made for other forms of screen time, including social media use, time spent on Apps, and online or mobile device activities in general. Yet, rather than focusing exclusively on screen time, other elements may be considered such as content, platform (context), and the device (media/technology) with which digital technology is used, as well as, most importantly, who is playing and why.

Digital technology itself provides the means to acquire information on these issues. Passive sensing includes data gathered in the background without any user involvement. The content of screen-based activity may be passively logged (App usage on a smartphone or the browser timeline), allowing for investigation of the context using, for example, the sensors of a mobile phone (accelerometer, location, Bluetooth, light sensors). Moreover, mental health indicators may be assessed using sensor data [9]. In addition, the experiences of adolescents in the process of interacting with digital technology may be assessed in daily life using ecological momentary assessment, which provides the means to characterize the immediate effects of media use in a naturalistic setting.

The need to involve different stakeholders in the development of research on the use of digital technologies and mental health cannot be overemphasized, including insights from industry experts but, most especially, children and adolescents growing up in the digital age who have considerable expertise at the cutting edge of digital experience. Their perspectives should be sought in defining screen time and in other aspects of research activity on this topic. In the end, this would heighten the pertinence of results and facilitate knowledge transfer into public health practice, providing guidance for the safe and effective integration of digital technologies into existing mental health services or educational settings.

A number of solutions thus follow from findings of the Orben review and the present discussion. First, in order to move this field of research forward we need to focus on user engagement for better understanding of the overall complexities of digital technology use, particularly in relation to screen time and its impact on child and adolescent mental health as a "complex problem." Second, more than a simple dose-response relationship as described by Paracelsus, research needs to move away from linear research designs, including limited definitions of screen time, in favor of approaches that are nonlinear, heterogeneous, open and adaptable. Particular attention must be given to user characteristics, digital divide and platform as well as the effects of possible confounding variables and the context in which user behaviour and interactions take place. This would include environmental and sociocultural factors (e.g., gender, social class, race, ethnicity, lifestyle), as well as the interconnected influences of larger socioeconomic structures (e.g., health care and social welfare systems, and labor markets). It is therefore necessary to characterize this exposure [10] and to investigate causal relationships through longitudinal studies.

\section{Conclusion}

Investigations of the content, context and motives for digital technology or media use are well within the reach of current research. This commentary suggests the need for a paradigm shift regarding the operationalization of screen time based on input from various stakeholders and new approaches to the study of digital technology use. Children and adolescents, as the primary consumers of digital technologies, are well positioned to provide input that will lead researchers to discover new perspectives on research design, aims and objectives as well as innovative methodological approaches. 
To unpack the interaction between users, devices, and context/environment, researchers need to embrace models and methodologies that allow for nonlinearity, heterogeneity, openness, and adaptation. While involving detailed and sometimes tedious experimental work, approaches that recognize the complexity of associations between digital technology and mental health hold great promise for advancement of the field.

Acknowledgements We would like to thank Judith Sabetti for revising earlier versions of this manuscript.

\section{Compliance with ethical standards}

Conflict of interest The authors declare that there is no conflict of interest.

\section{References}

1. Orben A (2020) Teenagers, screens and social media: a narrative review of reviews and key studies. Soc Psychiatry Psychiatr Epidemiol. https://doi.org/10.1007/s00127-019-01825-4

2. Dickson K, Richardson M, Kwan I, Macdowall W, Burchett H, Stansfield C, Thomas J (2019) Screen-based activities and children and young people's mental health and psychosocial wellbeing: a systematic map of reviews. EPPI-Centre, Social Science Research Unit, UCL Institute of Education, University College London, London

3. Tapscott D (2008) Grown up digital. McGraw-Hill Education, Boston

4. Livingstone S (2009) Children and the Internet. Polity Press, Oxford

5. Farhangi S (2012) Reality is broken to be rebuilt: how a gamer's mindset can show science educators new ways of contribution to science and world?. Springer, New York

6. McGonigal J (2011) Reality is broken: why games make us better and how they can change the world. Penguin, London

7. Dill KE, Dill JC (1998) Video game violence: a review of the empirical literature. Aggress Violent Behav 3(4):407-428

8. Ferguson CJ (2007) Evidence for publication bias in video game violence effects literature: a meta-analytic review. Aggress Violent Behav 12(4):470-482

9. Trifan A, Oliveira M, Oliveira JL (2019) Passive sensing of health outcomes through smartphones: systematic review of current solutions and possible limitations. JMIR mHealth uHealth 7(8):e12649

10. Guloksuz S, van Os J, Rutten BP (2018) The exposome paradigm and the complexities of environmental research in psychiatry. JAMA Psychiatry 75(10):985-986 